\title{
Review of: "Meibomian gland dysfunction is highly prevalent among first-time visitors at a Norwegian dry eye specialist clinic"
}

Melissa Toyos

Potential competing interests: The author(s) declared that no potential competing interests exist.

This is well written and well presented presentation but I do not believe it adds to the body of literature. Because the systems of dry eye are intertwined: aqueous deficiency goblet cell and mgd, I would expect all subjects with dry eye symptoms presenting to a specialized dry eye clinic to have some degree of mgd. The questionnaires are slightly interesting but already validated and no signals emerge in this study to change the way we use questionnaires to correlate signs or symptoms of dry eye 